\title{
Esofagectomia torácica parcial no tratamento de leiomioma em esfíncter esofágico inferior de cão
}

\author{
Partial thoracic esophagectomy in the treatment of leiomyoma in the lower \\ esophagealsphincter of dogs
}

\section{Thadeu Mourão Pinto' ${ }^{1}$, Lucila Maria de Almeida Lopes², Tuane Nerissa Alves Garcez², Rafael Stedile², Carlos Afonso de Castro Beck ${ }^{3}$ \& Emerson Antonio Contesini ${ }^{3}$}

\begin{abstract}
RESUMO
Tumores esofágicos em cães são raros e dentre estes, o leiomioma é o mais relatado. Os sinais clínicos mais comuns são disfagia, regurgitação e emaciação. Foi atendido no Hospital de Clínicas Veterinárias (HCV) da Universidade Federal do Rio Grande do Sul (UFRGS) um cão da raça Dálmata, macho, oito anos, apresentando sialorreia, regurgitação, vômito, algia abdominal e emaciação. No exame endoscópico visibilizou-se massa tumoral próxima ao esfíncter esofágico inferior e fundo gástrico, e foram coletadas amostras para análise histopatológica, obtendo-se resultado compatível com leiomioma esofágico e hiperplasia gástrica. Realizou-se ressecção esofágica e anastomose esôfago-gástrica. Decorridos três dias de pós-operatório o animal foi a óbito. Na necropsia não foram observadas alterações macroscopicamente significativas e microscopicamente sugeriu-se insuficiência renal. Este relato tem o objetivo de apresentar um caso de leiomioma em esfíncter esofágico inferior em um cão, no qual a terapêutica adotada foi a exerese tumoral por toracotomia.
\end{abstract}

Descritores: canino, cárdia, neoplasia, oncologia, toracotomia.

\begin{abstract}
Esophageal tumors are rare in dogs, among these, leiomyoma is the most reported. The most common clinical signs are dysphagia, regurgitation and emaciation. An 8-year-old, Dalmatian male dog was referred to the Hospital de Clínicas Veterinárias (HCV), Universidade Federal do Rio Grande do Sul (UFRGS) presenting drooling, regurgitation, vomiting, abdominal pain and emaciation. Endoscopic examination revealed a growth near the lower esophageal sphincter and gastric fundus, and samples were collected for histopathological examination. The results were consistent with esophageal leiomyoma and gastric hyperplasia. Esophageal resection and esophageal-gastric anastomosis was conducted. Three days post-operative the animal died. At necropsy no changes were observed macroscopically, and microscopically significant renal insufficiency was suggested. This report aims at presenting a case of leiomyoma at lower esophageal sphincter in a dog, in which the adopted therapy was tumor exeresis by thoracotomy.
\end{abstract}

Keywords: canine, cardia, cancer, oncology, thoracotomy.

${ }^{1}$ Programa de Pós-graduação em Ciências Veterinárias (PPGCVET), Universidade Federal do Rio Grande do Sul (UFRGS). ${ }^{2}$ Hospital de Clínicas Veterinárias (HCV) - UFRGS. ${ }^{3}$ Departamento de Medicina Animal, Faculdade de Veterinária (FaVet), UFRGS, Av. Bento Gonçalves no. 9090, CEP 91540-000 Porto Alegre, RS, Brasil. CORRESPONDÊNCIA: E.A. Contesini [emerson.contesini@ufrgs.br; Fax: + 55 (51) 3308 7305]. 


\section{INTRODUÇÃO}

Tumores de esôfago são raros em cães e gatos $[2-4,6,10]$. Dentre os diferentes tipos de tumores esofágicos incluem-se leiomioma, leiomiossarcoma, osteossarcoma, fibrossarcoma, carcinoma de células escamosas e plasmocitoma [10]. Apesar de o leiomioma ser descrito como a neoplasia de maior frequência, esta geralmente é considerada um achado casual $[2,4,6,10]$, localizando-se mais comumente no esôfago distal, em especial no esfíncter esofágico inferior [10].

Tumores menores que cinco centímetros podem ser assintomáticos $[4,10]$. A sintomatologia mais comum cursa com disfagia, regurgitação e emaciação $[2,4,6,10]$. O diagnóstico é sugerido através dos sinais clínicos e dos achados de radiografia, esofagoscopia e toracotomia [10], sendo a endoscopia indispensável para determinar a localização da massa e as condições da mucosa [4]. O tratamento de eleição para animais sintomáticos é a cirurgia [4,6, $8,10]$, a qual geralmente tem efeito curativo $[8,10]$.

Este relato tem o objetivo de apresentar um caso de leiomioma em esfíncter esofágico inferior em um cão, no qual a terapêutica adotada foi a exerese tumoral por toracotomia.

\section{RELATO DE CASO}

Um cão de oito anos de idade, macho, da raça Dálmata, pesando $19 \mathrm{~kg}$, foi atendido no Hospital de Clínicas Veterinárias da Universidade Federal do Rio Grande do Sul, com histórico de sialorreia, regurgitação, vômito, algia abdominal e emaciação. $\mathrm{O}$ exame radiográfico simples apresentou aumento de radiopacidade na região de esôfago caudal. Na endoscopia visibilizou-se massa tumoral próxima ao esfíncter esofágico inferior e fundo gástrico. Foram coletadas amostras para análise histopatológica e o resultado foi leiomioma esofágico e hiperplasia gástrica. A análise hematológica indicou linfopenia e os exames bioquímicos (ALT e creatinina) apresentavam-se dentro dos parâmetros normais para a espécie.

Iniciou-se tratamento clínico, com fluidoterapia utilizando solução de ringer lactato adicionado à glicose $50 \%$ e fructose $2,5 \mathrm{~g}(60 \mathrm{ml} / \mathrm{kg} / 24 \mathrm{hs})$; antibioticoterapia com enrofloxacina (5 mg. $\mathrm{kg}^{-1}, \mathrm{IM}$, BID) e metronidazol (10 mg. $\mathrm{kg}^{-1}$, IV, TID); cloridrato de tramadol (3 mg.kg-1 $, \mathrm{SC}, \mathrm{TID})$; ranitidina (2 mg. $\mathrm{kg}^{-1}$,
SC, TID); cloridrato de metoclopramida (0,5 mg. $\mathrm{kg}^{-1}$, $\mathrm{SC}, \mathrm{TID})$ e ondansetrona ${ }^{1}\left(0,3 \mathrm{mg} \cdot \mathrm{kg}^{-1}, \mathrm{IV}, \mathrm{TID}\right)$.

Devido à desnutrição protéico-calórica e à disfagia, procedeu-se a colocação de sonda nasogástrica que foi removida em menos de 24 horas, devido à disfunção esofágica do animal. Optou-se então pela sonda de gastrostomia via endoscópica percutânea. Após, introduziu-se ao tratamento sucralfato ${ }^{2}(25$ mg.kg ${ }^{-1}$ via sonda gástrica, BID) e omeprazol ${ }^{3}$ (1 $\mathrm{mg} \cdot \mathrm{kg}^{-1}$, via sonda gástrica, SID). $\mathrm{O}$ animal foi alimentado através da sonda, cinco vezes ao dia, com $100 \mathrm{~mL}$ de ração pastosa comercial, permanecendo os episódios de regurgitação.

Uma semana após a colocação da sonda de gastrostomia o paciente ainda apresentava sinais clínicos como regurgitação e emese, porém com menor frequência. Optou-se pela cirurgia convencional para remoção da neoplasia.

Como medicação pré-anestésica foi utilizada metadona $^{4}\left(0,2 \mathrm{mg} \cdot \mathrm{kg}^{-1}, \mathrm{IM}\right)$, a indução foi feita com propofol (4 mg. $\left.\mathrm{kg}^{-1}, \mathrm{IV}\right)$, e em seguida o animal foi intubado e mantido em plano anestésico com inalação de isoflurano em oxigênio $100 \%$ com circuito anestésico semi-fechado. Como profilaxia antimicrobiana foi utilizada ampicilina (20 mg.kg-1, IV) 30 minutos antes do início do procedimento cirúrgico.

Após a tricotomia cuidadosa da região, o animal foi colocado em decúbito lateral esquerdo e procedeu-se a antissepsia e a delimitação da área cirúrgica com campos esterilizados. Foi realizada toracotomia ao nível do oitavo espaço intercostal direito, sendo identificado e isolado o esôfago torácico, desde o terço médio até a sua porção distal, ao nível do cárdia. Com visualização direta observou-se que a massa tumoral localizava-se no esfíncter esofágico inferior, e media aproximadamente $4 \mathrm{~cm}$ de comprimento (Figura 1).

Foi necessária a incisão do diafragma para exposição do estômago. Realizou-se a ressecção esofágica e anastomose esôfago-gástrica em plano único extramucoso término-terminal, com fio poliglactina 910 (3.0) em pontos simples separados (Figura 2). A tensão foi aliviada por miotomia circunferencial. Foi mantida a sonda de gastrostomia e o tratamento clínico anteriormente instituído.

Decorridos três dias de pós-operatório, o animal foi a óbito. Na necropsia, macroscopicamente não foi observado deiscência de sutura da anastomose 
esôfago-gástrica e não havia sinais compatíveis com peritonite no local em que a sonda de gastrostomia estava fixada (Figura 3). Microscopicamente foi sugerida insuficiência renal.

\section{DISCUSSÃO}

O leiomioma é o tumor benigno esofágico mais comum, e geralmente é um achado casual [2$4,6,10]$. Apresenta-se mais comumente localizado no esfíncter esofágico inferior [10], como descrito no relato. Tumores com menos de $2 \mathrm{~cm}$ de diâmetro raramente cursam com sintomatologia [10], no entanto, o animal apresentava nódulo medindo $4 \mathrm{~cm}$ de diâmetro e sinais clínicos como disfagia, regurgitação, vômito, algia abdominal e emaciação, os quais são

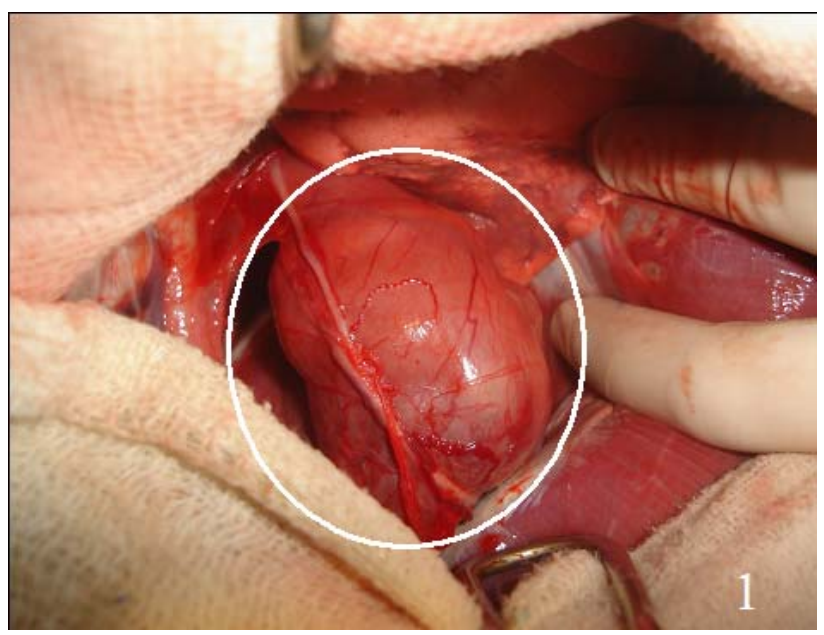

Figura 1. Canino, macho, dálmata, oito anos de idade, apresentando aumento de volume próximo a esfíncter esofágico inferior (círculo).

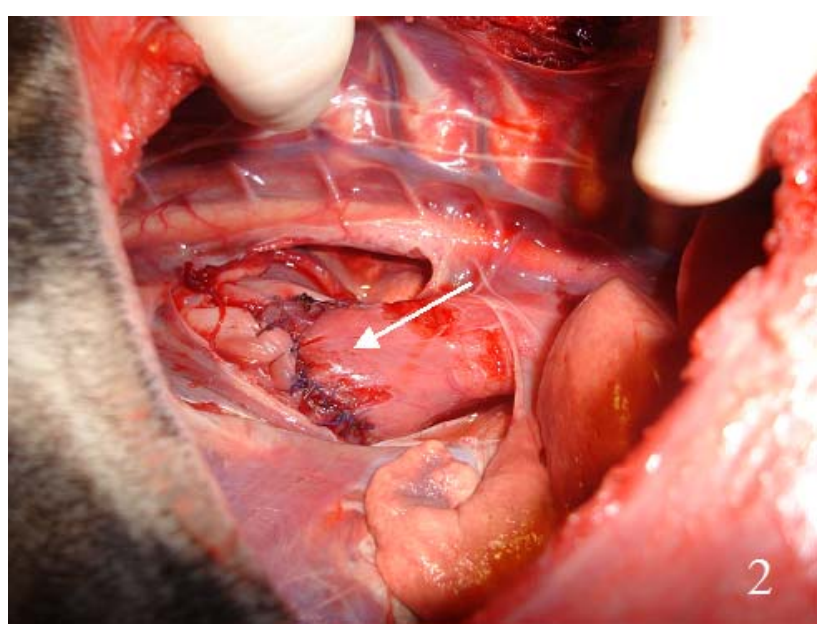

Figura 2. Aspecto da anastomose esôfago-gástrica em plano único extramucoso término-terminal, com fio poliglactina 910 (3.0) em pontos simples separados (seta). quadros clínicos compatíveis com os relatados na literatura $[2,4,6,10]$.

O diagnóstico é sugerido através da sintomatologia e os exames de imagem como radiografia e endoscopia são indispensáveis para conclusão do diagnóstico $[4,6]$. A biópsia endoscópica quando realizada deve ser profunda, possibilitando a ocorrência de hemorragia, perfuração e aderência da mucosa, por isso deve ser evitada [6,8]. Foi necessária a realização de biópsia incisional por endoscopia, no entanto não foram observadas intercorrências durante o procedimento.

Devido à desnutrição protéico-calórica do animal e à disfagia, procedeu-se a colocação de uma sonda nasogástrica que foi removida em menos de 24 horas, pois o animal persistiu com os episódios de regurgitação e vômito. Em casos de disfunção esofágica e vômitos persistentes o uso desta sonda é contra-indicado [5]. Optou-se então pela sonda de gastrostomia endoscópica percutânea. Recomendase o uso de nutrição enteral por sonda gástrica, pois esta demonstrou ser um método eficiente devido à ausência de movimentos de deglutição e de atrito do bolo alimentar sobre a ferida cirúrgica [1,9]. Uma das complicações dessa sonda é a peritonite por extravasamento de conteúdo gástrico devido à formação inadequada de aderências no local de fixação da sonda [5], o animal do relato não apresentou sinais de peritonite.

Uma semana após a colocação da sonda de gastrostomia o paciente ainda apresentava sinais clí-

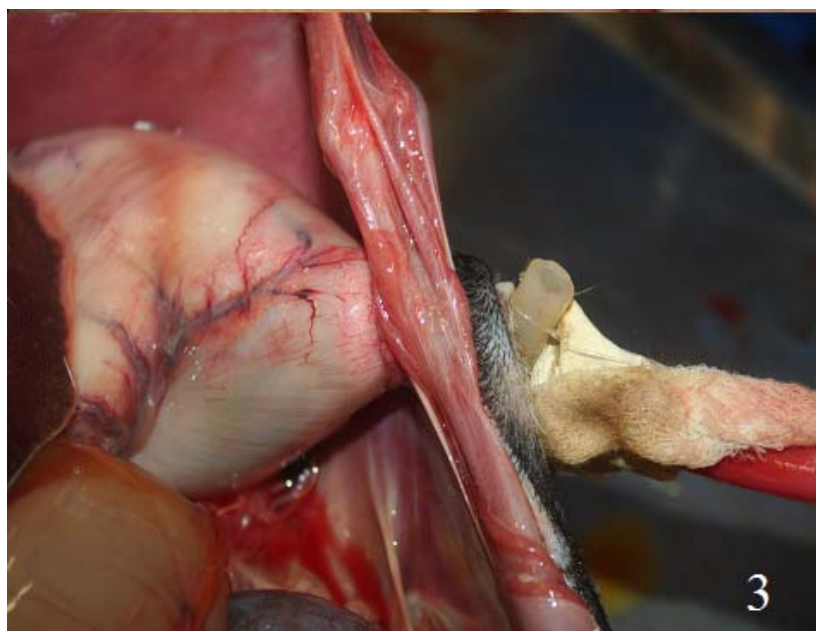

Figura 3. Canino, macho, raça Dálmata, oito anos de idade. Visualização macroscópica do local de fixação da sonda de gastrostomia no exame post-mortem. Notar ausência de sinais de peritonite no local. 
nicos como regurgitação e emese, provavelmente devido à localização da massa tumoral, porém com menor frequência. Optou-se pela cirurgia convencional para remoção neoplásica e com a visualização direta observou-se que a massa tumoral localizavase no esfíncter esofágico inferior. A literatura indica a ressecção esofágica por toracotomia nos casos em que o tumor é grande e na existência de sintomas como disfagia [2], a qual foi adotada no caso apresentado.

Ressecções de mais de 3 a $5 \mathrm{~cm}$ do esôfago aumentam os riscos de deiscência, sendo necessário em alguns casos técnicas de substituição do esôfago [5]. Apesar de a extensão da lesão ser de aproximadamente $4 \mathrm{~cm}$ de diâmetro, não foi utilizado técnica de substituição do esôfago, sendo assim, houve a necessidade de aliviar a tensão da anastomose com miotomia circunferencial [5]. Essa técnica não diminuiu a possibilidade de deiscência, além de ter promovido alte- rações deletérias na cicatrização da anastomose [9].

O prognóstico de leiomioma esofágico é bom, e normalmente a cirurgia quando necessária tem efeito curativo [10]. O animal do relato apresentou uma grave deficiência nutricional, que geralmente aumenta quando o animal apresenta disfunção gastrintestinal, efeito colateral de medicações, medo e/ou dor. O catabolismo significativo de proteína durante a inanição reduz seriamente a sobrevivência em longo prazo [7]. No exame post-morten macroscopicamente não foi observado deiscência de sutura da anastomose esôfagogástrica, sendo a cirurgia eficaz no período observado.

\section{NOTAS INFORMATIVAS}

${ }^{1}$ Cloridrato de Ondansetrona, Hypofarma, Ribeirão das Neves, Minas Gerais.

${ }^{2}$ Sucralfilm, Laboratório EMS, Hortolândia, São Paulo. ${ }^{3}$ Pratiprazol, Prati Donaduzzi \& CIA LTDA, Toledo, Paraná. ${ }^{4}$ Mytedom, Cristália, Itapira, São Paulo.

\section{REFERÊNCIAS}

1 Barcellos H.H.A., Silva Filho A.P. \& Beck C.A. 2000. Influência de três vias de fornecimento de dietas pós-operatórias na cicatrização cervical em cães. Brazilian Journal of Veterinary Research and Animal Science. 37(5): 184-193.

2 Beltrán J.B., Moreno J.M.Z., Rodríguez J.M.Z. \& Sotelo C.S. 2005. Leiomioma del esôfago y su resección endoscópica transhiatal. Informe de um caso. Associón Mexicana de Cirugía Endoscópica, A.C. 6(3): 135-140.

3 Bolzán H., Spatola J. \& Chiarenza C. 2005. Resección endoscópica de um leiomioma de esôfago mediante la ligadura com banda elástica. Acta Gastroenterologica Latinoamericana. 35(3): 165-168.

4 Estrada D.P., Matteoda M., Belaunzarán A. \& Calvo S. 2005. Leiomioma esofágico. Revista Del Hospital Privado de Comunidad. 8(2): 17-20.

5 Kyles A.E. 2007. Esôfago. In: Manual de cirurgia de pequenos animais. 3.ed. São Paulo: Manole, pp.573-592.

6 Lorencetti R.R.G., Goes J.F.V., Mattion E.A., Módena S.F. \& Paula R.A. 2005. Exérese de Leiomioma Esofágico por Toracotomia. Revista do Colégio Brasileiro de Cirurgia. 33(1): 55-56.

7 Mauldin G.E. \& Davidson J.R. 2007. Suporte Nutricional de Cães e Gatos Hospitalizados. In: Manual de cirurgia de pequenos animais. 3.ed. São Paulo: Manole, pp.87-113.

8 Priego P., Lobo E., Rodríguez N.A., Olarte M.A.G., Oteyza J.P. \& Fresneda V. 2006. Surgical treatment of esophageal leiomyoma: an analysis of our experience. Revista Espanola de Enfermedades Digestivas. 98(5): 350-358.

9 Tannuri U, Tannuri A.C.A., Fukutaki M.F., Oliveira M.S., Muio V.M.F. \& Massaguer A.A. 1999. Effects of circular myotomy on the healing of esophageal suture anastomosis: an experimental study. Revista do Hospital das Clínicas. 54(1): 9-16.

10Thomson M. 2007. Trato Alimentar e Pâncreas. In: Manual de cirurgia de pequenos animais. 3.ed. São Paulo: Manole, pp.2368-2378.

11 Valadares R.C., Palhares M.S., Bicalho A.L.F., Turcheto Jr. C.R., Freitas M.D., Silva Filho J.M. \& Carvalho A.U. 2006. Aspectos clínicos e hematológicos em cães submetidos à fluidoterapia intravenosa, nutrição enteral e parenteral. Arquivo Brasileiro de Medicina Veterinária e Zootecnia. 58(4): 495-502.

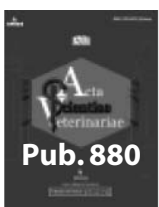

